\title{
REVISIONES
}

\section{Corporalidades y narrativas docentes: un dispositivo metodológico para la investigación y formación de profesores*}

\author{
Corporealities and Teachers narratives: a methodological device \\ for the research and development of teachers
}

\author{
Ana Arévalo V. ${ }^{a}$, Belén Fernández Ll. ${ }^{b}$, Felipe Hidalgo K. ${ }^{c}$, Yasna Lepe S. ${ }^{d}$, \\ Christian Miranda J. ${ }^{e}$, Mauricio Núñez R. ${ }^{f}$, Leonora Reyes J. ${ }^{g}$ \\ ${ }^{a}$ Universidad de Chile. Correo electrónico: anarevalov@ gmail.com \\ ${ }^{\text {b} U n i v e r s i d a d ~ d e ~ C h i l e . ~ C o r r e o ~ e l e c t r o ́ n i c o: ~ b e l e n f e r n a n d e z l l @ g m a i l . c o m ~}$ \\ c Universidad de Chile. Correo electrónico: felipehidalgok@gmail.com \\ d Universidad de Chile. Correo electrónico: tar_danza@yahoo.es \\ e Universidad de Chile. Correo electrónico: christian.miranda@u.uchile.cl \\ ${ }^{\mathrm{f}}$ Universidad de Chile. Correo electrónico: mauricio.nunez@u.uchile.cl \\ g Universidad de Chile. Correo electrónico: leoreyesj@gmail.com
}

\section{RESUMEN}

En este artículo se presenta un dispositivo metodológico narrativo-corporal, el cual pretende aproximarse a las subjetividades de un grupo de docentes del sistema escolar chileno.

En una primera parte se describen antecedentes contextuales y emergentes que dan origen al dispositivo propuesto. En un segundo apartado se presentan las características del dispositivo en cuestión para, posteriormente, analizar los aportes del mismo como instancia formativa e investigativa, tanto para los docentes como para el equipo involucrado en este proyecto.

Finalmente, se discuten los alcances que ha tenido este dispositivo para indagar en la experiencia subjetiva y en los procesos de formación colaborativa de los docentes que formaron parte del taller que esta iniciativa ofreció.

Palabras claves: Cuerpo, subjetividad docente, relatos de experiencia, formación de profesores

\section{ABSTRACT}

This article presents a narrative-body methodological device used to approach the subjectivity of teachers in the Chilean school system. In the first part some contextual background and the emergence of the creation of this device are presented. In a second section the main features of it are exposed to then analyse its contributions as a training and research analysis, both for teachers and researchers involved in this project. Finally, the possibilities of this device to investigate the subjective experience and the processes of collaborative training of teachers in the school system in Chile are discussed.

Keywords: Corps, teacher's subjectivity, narratives of teaching experience, teacher training

Este trabajo se enmarca en el desarrollo del Núcleo de Investigación "Subjetividad docente en Chile: voces y experiencias desde el profesorado del sistema escolar", Iniciativa Bicentenario JGM, Fondo de Investigación / Creación, INTERD-2-3 y los proyectos FONDECYT N ${ }^{\circ} 1140744, \mathrm{~N}^{\circ} 1140827$ y N 11130244 financiados por la Comisión Nacional de Investigación Científica y Tecnológica. 
Estudios Pedagógicos XLII, $\mathrm{N}^{\circ}$ 4: 223-242, 2016

CORPORALIDADES Y NARRATIVAS DOCENTES: UN DISPOSITIVO METODOLÓGICO PARA LA INVESTIGACIÓN

Y FORMACIÓN DE PROFESORES

\section{INTRODUCCIÓN}

La investigación científica nacional e internacional es consistente en señalar la relevancia de los docentes en el mejoramiento de la calidad educativa (Sander y Rivers, 1996; PRELAC, 2003; Robalino, 2005; UNESCO, 2008; Ávalos, 2013). Asimismo, la Organización para la Cooperación y el Desarrollo Económicos [OCDE] (2009) ha relevado la importancia que tiene atraer, formar y retener docentes de calidad. Esto ha conducido a que durante la última década los países han puesto mayor atención al trabajo de los profesores dado que, habiendo logrado avances significativos en cobertura educacional, es clave atender a la calidad de los procesos de enseñanza y aprendizaje que tienen lugar al interior del aula.

En este contexto de revalorización de la labor docente, las reformas educativas contemporáneas han buscado asegurar aprendizajes de calidad, mediante programas y proyectos caracterizados por: introducir cambios institucional esa nivel de gestión escolar; adoptar sistemas de medición de la calidad y evaluación de resultados de aprendizaje estandarizados; revisar los contenidos curriculares, priorizando áreas de alto impacto social y económico como matemáticas, lenguaje y ciencias, y profesionalizar la labor docente mediante incentivos de mercado (Vaillant, 2004; Assaél et al. 2011; Fardella y Sisto, 2015).

Sobre esto último, diversos estudios (Valdivia et al., 2003; Bravo, Peirano y Flack, 2006) evidencian que las reformas han incidido en una permanente necesidad de actualización y mayor responsabilización de resultados escolares por parte de los maestros. Debido a esto, sus condiciones laborales se han visto tensionadas, impactando tanto en su valoración social como a nivel subjetivo (Cornejo y Reyes, 2008). Asimismo, se cuestionan sus saberes en el ámbito disciplinario y pedagógico, lo cual se vería reflejado en bajos rendimientos escolares en áreas relevantes del currículum escolar (Miranda, Gysling, Rivera, Arancibia, y López, 2015), desvalorizando con ello saberes experienciales acumulados. Considerar esto último significa repensar desde enfoques alternativos la formación e investigación sobre la figura del docente (Reyes et al., 2014).

En armonía con lo último, un equipo de académicos de la Universidad de Chile, desde el año 2013, ha articulado líneas de investigación que abordan la temática del profesorado, con la finalidad de constituir un Grupo Interdisciplinario sobre este campo de estudio. Uno de sus propósitos principales ha sido la construcción de dispositivos metodológicos que permitan abordarla subjetividad docente, en una dimensión tanto formativa como investigativa, mediante la exploración de nuevos lenguajes basados en el trabajo corporal y narrativo.

De este modo, este artículo tiene por finalidad describir un dispositivo metodológico, para la formación e investigación con profesores, construido durante los últimos años por este equipo interdisciplinario y analizar los principales hallazgos generados luego de su implementación.

\section{ANTECEDENTES CONCEPTUALES}

\subsection{EL CUERPO}

Durante las últimas décadas, la categoría de cuerpo ha cobrado relevancia en el campo de las ciencias sociales y humanidades, llegando a considerarse como un elemento clave desde donde mirar los procesos históricos y socioculturales, así como los procesos de constitución y despliegue de los sujetos en las sociedades contemporáneas (Hancock et al., 2000, Le Breton, 2002). 
Si bien en la modernidad la representación dominante del cuerpo ha provenido del discurso biomédico (Hughes, 2000), este ha sido objeto de estudio en las ciencias sociales, definiéndolo como un fenómeno social, cultural e histórico, inserto en constantes tramas de sentido y significaciones. Pasando desde una etapa (s. XIX) en la cual las ciencias sociales no lograban darle mayor significación (Le Breton, 2002), ya durante el siglo XX se han desarrollado perspectivas ontológicas y epistemológicas diversas para estudiar el cuerpo y sus manifestaciones, siendo los principales enfoques aquellos adscritos a la fenomenología (Merleau-Ponty, 2002), el post-estructuralismo (Foucault, 1975, 2010; Turner,1989), el psicoanálisis (Freud, 1981; Lacan, 1985) y las teorías feministas (Butler, 2007).

En el ámbito educativo, según Mclaren (1997), la temática del cuerpo ha tendido a ser ignorada, imposibilitando con ello un trabajo de teorización de las prácticas educativas desde este ángulo de análisis, pese a la encarnación de valores culturales hegemónicos mediante la docilidad del cuerpo que ocurre en el espacio de la escuela (Foucault, 1975; Kirk, 1998; Evans, Davies y Wright, 2004). En la literatura especializada, a los fines de este estudio se destacan los trabajos sobre las relaciones y configuraciones entre la corporalidad y lugar-espacio de aprendizaje (Bánovcanová y Slavík, 2014), las didácticas específicas utilizando el cuerpo para el desarrollo de aprendizajes (Roth, 2014; Quay, Dickinson and Nettleton, 2003); las representaciones de los profesores y estudiantes respecto al cuerpo (Rich, 2010; Vander y Gard, 2014); las diversas teorías del cuerpo y movimiento humano, y su empleo en la educación física (Sergio, 1999; Le Boulch, 1971; Parlebas, 1981); las acciones performativas que son constitutivas de la identidad de los docentes (Vick y Martínez, 2011); y la importancia que le asignan los profesores al cuerpo para entender su quehacer (McWilliam y Taylor, 1996).

\subsection{LAS NARRATIVAS}

Las narrativas docentes en la investigación educacional han sido el resultado de un largo periplo que parte con los estudios pioneros en antropología y sociología de la Escuela de Chicago en los años 20 y que vuelve en nuestro tiempo a su punto de partida: el sujeto.

La noción que media la perspectiva narrativa es la de la escucha de la voz de los sujetos, entendida como aquella que es portadora de historias de vida. Ponerse a la escucha requiere apertura, sensibilidad, empatía, para así comprender lo que los otros han vivido. Esta tradición fue continuada en el mundo de la educación por investigadores que en los años 80-90, manifestaron la necesidad política y epistemológica de ponerse a la escucha de los sujetos docentes (Elbaz, 1991). Goodson (1992) desarrolla estudios de las biografías docentes, bajo la premisa de que la vida de los profesores tenía mucho que aportar para comprender su trabajo. En esta misma línea, Connelly y Clandinin (1994), bajo la metáfora de la reconstrucción, proponen estudios de las narrativas docentes considerando elementos biográficos de la formación de profesores. Finalmente, Bajtin (1995) considera las historias de vida de los docentes en todas sus esferas, bajo el supuesto que vida ordinaria de sujetos es una dimensión relevante de profundizar.

Sobre lo anterior, Núñez (2015) sostiene que el espacio para la emergencia de la voz docente, para su escritura y su puesta en comunidad es aún, en la perspectiva temporal, un espacio político no ganado. Incluso, testimoniando el éxito en la propuesta, siguen siendo, en términos globales, experiencias marginales, que atraen, que seducen, que pueden convencer por su alto impacto, pero que no se condicen con las políticas mundiales vigentes en educación, orientadas por el enfoque de escuela eficaz. 
Respecto a los dispositivos metodológicos para profesores que han recogido lo corporal y/o narrativo, se puede nombrar, por ejemplo, el trabajo en la formación de profesores de la Universidad de Valladolid, los cuales han creado cinco bloques de contenidos, en donde se trabaja lo corporal desde una perspectiva fenomenológica (cuerpo vivido). Aquí destaca el quinto bloque llamado "El cuerpo del docente", referido a su salud laboral, el carácter comunicativo del cuerpo, las relaciones personales y emociones en el quehacer de estos y el yo corporal, entendido este último como el proceso de configuración corporal que han recorrido los profesores durante sus vidas (Martínez, 2013).

En relación al trabajo con narrativas, que abordan desde lo personal a lo profesional, se puede reconocer un camino más perfilado que el de las corporalidades, por estar un poco más cercano a las racionalidades y la cognición tradicionales. En este punto resulta evidente que el trabajo desde las narrativas, aun cuando aborda las subjetividades e intersubjetividades en el seno de una comunidad escritural, sigue siendo menos rupturista que el trabajo con la corporalidad para los enfoques más ortodoxos de investigación. Aquí es pertinente poner en relato las vivencias de los profesores, de modo tal de transformarlas por el ejercicio reflexivo explícito, en experiencias docentes. El National Writing Project en los Estados Unidos posee una historia de más de cuarenta años que ha sido sistematizada por Lieberman (Lieberman \& Wood, 2003). En Argentina, el Movimiento Memoria Docente y Documentación Pedagógica (Suarez, 2007; 2007b) viene trabajando desde hace más de una década en la sistematización y construcción de un repertorio de historias docentes. Lo central y común de ambas experiencias es la de la existencia de comunidades de sostén y de escritura, que posibilitan el desarrollo de las narraciones como resultado de la complejización de la mirada individual mediada por la crítica de una comunidad de pares.

Otras experiencias relevantes a los fines de este estudio son la Expedición Pedagógica Nacional en Colombia (Suárez, H., 2001) y los trabajos sobre escritura profesional docente de Morisse, Lafortune y Cros $(2009 ; 2011)$ y de Reis y Climent (2012), en el ámbito francófono y español respectivamente.

Finalmente, una propuesta que avanza en la convergencia entre narración y cuerpo es la desarrollada por Márquez, Padua y Prados (2015), en la Universidad de Almería. Esta, utilizando una metodología basada en el uso de biografías escolares, observa cómo a partir de actividades de conciencia y expresión corporal un grupo de estudiantes de pedagogía comprenden los significados emocionales que están presentes en el quehacer docente y que son constitutivos de la identidad profesional.

\section{DISPOSITIVO: TALLER DE NARRATIVAS Y CORPORALIDAD DOCENTE}

El Grupo Interdisciplinario ya mencionado, desde distintas trayectorias y experiencias investigativas se ha orientado a la comprensión del sujeto y la subjetividad del docente actual. Este foco surge para comprender la experiencia y el trabajo de la enseñanza en el marco de un sistema escolar que no escucha sus voces, y por el afán de generar innovaciones tanto en el plano investigativo como en la formación de los docentes, que permitan trabajar con los profesores y sus experiencias más que sobre ellos. En este sentido, se asume un giro epistemológico que sitúa al grupo en un enfoque colaborativo en el cual un dispositivo metodológico articula tanto los intereses de investigación como los de formación (Desgagné, 1997; Desgagné et al., 2001). 
Otro aspecto asociado a la idea de colaboración se percibe tras la articulación y diálogo de diversas miradas provenientes de la pedagogía, las ciencias de la educación, las humanidades y las ciencias sociales.

No obstante, la innovación resultado del foco explicitado, tuvo relación con la creación de un dispositivo metodológico, el cual es nutrido con la experiencia investigativa de los integrantes de su equipo, como también con las diversas aproximaciones y experiencias prácticas de los profesores del sistema escolar. Así, luego de una fase de diálogo y trabajo colaborativo, entre el 2014-2015, se creó un dispositivo dirigido a profesores del sistema escolar chileno, llamado Taller de expresión, corporalidad y narrativa docente (en adelante Taller), cuyos dos propósitos fueron:

a) Trabajar el movimiento expresivo del cuerpo como un dispositivo de exploración e investigación individual y colectiva, que promueva en los y las docentes la valoración y visibilización del cuerpo como una herramienta de sensibilidad e inteligibilidad y por ende, de aprendizaje, expresión, comunicación y creación.

b) Investigar, a través de la escritura de relatos de experiencia, los significados y sentidos que los docentes viven en la cotidianeidad de su trabajo y que han sido relevados desde lo corporal.

Se buscaba en dicha instancia articular ambos lenguajes a fin de complementarse, nutrirse y dar cuenta, en definitiva, de una misma experiencia docente en el marco de las condiciones de su trabajo.

La duración del Taller fue de seis sesiones, de 2,5 horas cronológicas cada una, y se efectuó durante las dos primeras semanas del mes de enero del año 2015. En ellas, participaron veinte docentes en servicio, en conjunto con los integrantes del Grupo Interdisciplinario en las actividades del mismo.

Durante el primer semestre del 2015 se procedió a reunir todo el material producido a través de notas de campo de los investigadores, filmaciones del Taller y los relatos finales que produjeron los profesores participantes. Luego de aquello, y de generar los primeros análisis del material recolectado, se realizó un encuentro de retroalimentación con los participantes para realizar un diálogo respecto a la experiencia vivida y poner en discusión los elementos emergentes, convergentes y divergentes de los primeros análisis.

Posteriormente, durante el segundo semestre del año 2015 se procedió a analizar en profundidad la experiencia del Taller, poniendo especial énfasis en los significados e interpretaciones que emergían desde el análisis corporal y narrativo.

\section{LAS COMPRENSIONES EMERGENTES DEL TALLER}

\subsection{DISPOSICIONES Y REPRESENTACIONES}

En el momento inicial del Taller se recogieron impresiones en relación a las disposiciones y representaciones de los docentes en torno a la convocatoria. Los participantes manifestaron sorpresa a la vez que disposición de apertura en relación al contenido de la convocatoria, con la idea de buscar algo diferente a la oferta predominante en la formación continua. La 
participación previa de algunos de ellos en instancias de trabajo corporal y escritural favoreció su disposición frente a la propuesta.

En relación a la dimensión corporal, los docentes participantes manifestaron la inquietud por el trabajo con el cuerpo en su relación con los estudiantes. Esto en cuanto a una común tensión entre su convicción y constatación acerca de que la corporalidad está asociada, en su vivencia en la escuela, a las relaciones afectivas, pero, a la vez es controlada, vigilada y rigidizada. $\mathrm{Al}$ decir de una de las participantes:

Soy bastante afectiva (con) mis alumnos... pero con todo este tema de la pedofilia se nos indica que, por favor, si un alumno te va a abrazar dile que no...Entonces, es súper chocante[...] cuando uno pasa por la sala lo ideal es que los alumnos estén todos sentados derechitos ...el cuerpo tiene que estar ahí encasillado, noventa minutos quieto y a veces ni yo estoy noventa minutos quieta... Entonces, hay una cosa con el cuerpo que es como una guerra constante entre el quehacer docente ...y las necesidades del cuerpo.

El cuerpo se percibe como "olvidado" en la experiencia vital docente, una dimensión no presente en su actuar cotidiano. Se lo ve también como el lugar donde se ejerce opresión sobre el/la profesor/a, pero a su vez se lo concibe, desde la creencia, poseedor de un potencial liberador o contra-hegemónico, como lo manifiesta el profesor:

...así como el profesor ideológicamente cumple mandatos y también tiene la posibilidad de generar una contra cultura, también el cuerpo es el campo donde esa opresión se imprime, y por lo tanto también tiene ese potencial contra hegemónico por decirlo de alguna forma.

En relación a las disposiciones y representaciones en torno a la dimensión narrativa, algunos docentes participantes las asociaron a un modo de desarrollo personal y profesional, vinculado a una práctica de escritura que se ha perdido, que se valora y que se desea restituir. Un docente, por ejemplo, llegó a referirse a este enfoque como una poderosa herramienta de trabajo:

la narrativa me interesa bastante porque, por una parte, siento la necesidad de tener elementos teóricos y metodológicos para reflexionar esta experiencia (docente)... por lo complejo que me ha tocado vivir.

Por otro lado, existe una diversidad de modos de disponerse para este tipo de trabajo:

[...] necesito hacerme más consciente de la importancia del cuerpo como de la narrativa, porque cotidianamente, la realidad demanda darse tiempo para uno para poder entender al otro, sino uno se bloquea.

Finalmente, en relación a un primer ejercicio corporal, cuya finalidad era la presentación personal de los asistentes, se revela la capacidad de los docentes de observarse y de disponerse en relación al otro/a, a pesar de reconocer la dificultad que ello conlleva. Así también fueron recurrentes las expresiones de empatía o percepción emotiva. En esta 
primera experiencia colectiva, ya se percibe la disposición corporal del otro: ver al otro, esto es, un modo de captación corporal que contiene en sí una dimensión reflexiva, y lo es en tanto logra captar aspectos diferenciadores (individualizadores) de quién se tiene delante:

[...] lo que me llegó de mi colega es lo pausado, de corporalidad entregada, relajada... buena onda; “... Lo que yo vi de ella es que es una persona muy feliz, y muy jovial, y está muy contenta con la vida [...] la vi tranquila, suave, acogedora.

Por último, en las sesiones se desarrolló un ejercicio de lectura colectiva de un relato de experiencia. Luego se les pidió a los docentes participantes responder a la pregunta ¿por qué se escribe? Las respuestas, en general, se centraron en la toma de conciencia. Por ejemplo, una docente señala que:

la escritura ayuda a darse cuenta... te hace ser consciente de lo que haces, a no ser tan autómata, es un cable a tierra,...es quitar la cortina, es como quitar un obstáculo que impide ver la realidad tal cual.

\subsection{DEL CUERPO A LA ESCRITURA}

El Taller continúa con una fase clave del proceso: el tránsito "del cuerpo a la escritura”. Se organiza según dos momentos: primeramente, se plantea un ejercicio corporal en que se desarrollan los conceptos de identidad, conciencia corporal y emocionalidad, seguido de un ejercicio narrativo en torno a la pregunta ¿qué me comunica el cuerpo en este momento de mi vida docente? Aquí se espera la escritura individual de un relato en torno a un episodio vivido en el contexto laboral que involucre la dimensión corporal.

El ejercicio corporal se orientó hacia la toma de conciencia de los cuerpos en el espacio, a través del trabajo colectivo y colaborativo. Los comentarios y reflexiones posteriores sobre el significado de este ejercicio, para cada uno, se jugó en relación a la retribución que recibieron del compañero/a al sentir su contacto y la conciencia explícita de que el cuerpo revela quienes somos. Un participante señaló:

me di cuenta de mi postura de caminar que es media tensa, y también creí percibir en el otro la postura tensa. Percibí en mi compañera dónde está la tensión... el cuerpo dice altiro, es acusete totalmente, no se queda callado, por más que uno trate de mentir.

Además, emerge el reconocimiento de la rigidez corporal como reflejo del miedo a sentirse vulnerables y como búsqueda de un modo de estar seguros:

me llamó la atención en el ejercicio de la caminata, como ésta proyecta ese miedo que tenemos a sentirnos vulnerables. Uno cuando camina expresa ese deseo de sentirse seguro en un lugar, yo lo expresé en lo rígido de mis rodillas...y aquí en mi compañera, en tener los brazos rígidos.

Por otro lado, la presencia del otro que lee lo que otro escribe, ve lo que otro no ve, ve lo que delata el cuerpo: 
Estudios Pedagógicos XLII, $N^{\circ}$ 4: 223-242, 2016

CORPORALIDADES Y NARRATIVAS DOCENTES: UN DISPOSITIVO METODOLÓGICO PARA LA INVESTIGACIÓN

Y FORMACIÓN DE PROFESORES

me di cuenta gracias a mi compañera de cosas que no sabía, una postura con los brazos, no había tomado conciencia de eso.

Finalmente, emerge la conciencia de estar blindados, protegidos. Uno de los participantes señaló:

tenemos adentro un microchip de autoprotección individualista, estamos muy blindados.

\subsubsection{Las violencias en el colegio}

La experiencia inaugural en torno al ejercicio de escritura constituyó un momento relevante para los participantes. Una atmósfera de silencio, atención y complicidad se apoderó del ambiente, tanto en el momento de la escritura individual como al dialogar en torno al ejercicio. Posterior a la lectura de algunos relatos se preguntó sobre qué dicen estos relatos y cómo son recibidos. Las temáticas oscilaban entre la agresión, el abuso a la infancia, la impotencia docente, la prohibición y la agresividad en el sistema escolar en general:

eran situaciones muy tristes, angustiantes, desagradables... Porque soy ser humano, tengo emociones, soy profesor y también quiero demostrar mi cariño a los alumnos y se me prohíbe hacerlo. La impotencia de no poder ayudar a la niña que ha sido abusada, qué hago yo como profe.

Los docentes participantes declararon identificarse con las experiencias de sus estudiantes y que fueron objeto de narración en cada uno de sus escritos. Estos, tenían como denominador común las formas de violencia contra la infancia que se presentan en el ámbito escolar y que si bien no surgen necesariamente en este, si se entrecruzan y profundizan. En el relato de uno de los docentes se señala que:

en el ámbito escolar esto se calla, es como un tabú, algo oculto que no hay que decir... todo hay que esconderlo debajo de la alfombra para que no se note y que esté el colegio impecable...lamentablemente los profesores no tenemos esta vía de escape de poder decir, de poder hablar, de poder expresar esto en un Consejo de profesores.

La falta de diálogo sobre las distintas formas en que se expresa la violencia encontraría su razón de ser, según la conversación posterior al ejercicio, en la primacía de la lógica técnico-pedagógica que focaliza las discusiones de la escuela en temas externos como el SIMCE, la evaluación docente y los cambios curriculares. Se omite con ello, los temas internos como los conflictos laborales y realidades complejas que se viven en el establecimiento, siendo preponderante una lógica de la apariencia, cuyo mensaje principal, según el relato de los participantes sería: "todo está bien, aquí no hay problemas".

No obstante, prevalece en los participantes del Taller el dilema respecto a cómo hacer frente a las violencias (físicas, verbales, psicológicas, entre otras) que sufren los niños y jóvenes. Y el dilema surge en tanto cómo se perciba a sí mismo el/la docente:-

porque... uno se compromete entero no una parte, es el cuerpo entero, cuerpo mente, cuerpo, sentimientos, emociones, alma, espíritu, todo. 
Es decir, justamente surge el dilema desde el entenderse como docente comprometido, lo cual es desafiado por una realidad de escuela "anulante" o "condenatoria" a tal implicancia, que genera sentimientos de "rabia" o "temor", o preguntas cuestionadoras: "¿por qué tengo que aguantar todo esto?”.

\subsubsection{El cuidado de los otros}

Desde un lugar más demarcado, en que se asume la tarea docente centrada en el vínculo humano, y en donde la exposición a situaciones emocionales dejan huellas, a veces dolorosas, una docente participante plantea un nuevo dilema. Cómo cuidarse si se es un docente identificado con un quehacer basado en el vínculo y cuidado de los demás:

yo trabajo con niños más pequeños que a veces les da una pataleta e igual te pegan, y si bien yo entiendo que es obvio que no es conmigo, uno igual está aceptando esa emoción tan potente que igual deja una huella en uno que es violenta. Y uno está diciendo 'ya, resisto esto', pero cómo al mismo tiempo comunico que me estoy cuidando, o ¿realmente me estoy cuidando?... Yo estoy exponiéndome a estas situaciones porque es desde el vínculo también, entonces se me generó esa pregunta de cómo poder ser profesor, cuidarme y ayudar... es súper complicado.

\subsection{DE LA ESCRITURA AL CUERPO}

El proceso prosigue hacia una nueva fase: la narrativa en la modalidad de escritura recursiva. El texto es elaborado de diversos modos: se retoma lo escrito, se lee, se comparte, se escucha, todo esto en el seno de pequeños grupos. La idea es abordar la textura del relato y para ello se propone orientar los comentarios que los pares puedan realizar, hacia la demanda de explicitación. Luego, con lo emergente de la discusión en torno a cada relato, se vuelve a los ejercicios con el cuerpo. De la narrativa a la corporalidad, y viceversa, se puede observar una dialéctica que se va haciendo presente a lo largo de todo el Taller.

\subsubsection{La corporalidad como juego escénico}

La propuesta de trabajo de la corporalidad se puede entender bajo la figura de un juego escénico. Al respecto, Gao Xingjian, ganador del premio Nobel de literatura del año 2000, refiriendo el juego escénico a la tradición del teatro oriental, lo presenta como una totalidad compleja en el que los movimientos, los gestos, las luces, los sonidos y el color, tratan de involucrar intelectual y emocionalmente al espectador. Existe una activación reflexiva de estos juegos que buscan expresar a través del cuerpo, momentos y emociones asociadas a los mismos. De este modo, se entiende en este trabajo que el juego escénico es también un relato y como tal es percibido e interpretado por los demás. Así, el juego escénico es construido y representado desde la experiencia de trabajo, desde la cotidianeidad, abriendo con ello la reflexión en torno al trabajo docente y su valoración. Ese relato, trazado en los gestos y movimientos, amplifica el registro de las emociones como al interior de una caja de resonancia.

La abstracción que permite el trabajo corporal no es posible en una propuesta escritural que opta por la narrativa más tradicional (no poética). Aquí no hay, necesariamente, lenguaje figurativo, no hay metáforas que permitan mostrar de otro modo lo vivido y las 
Estudios Pedagógicos XLII, N 4: 223-242, 2016

CORPORALIDADES Y NARRATIVAS DOCENTES: UN DISPOSITIVO METODOLÓGICO PARA LA INVESTIGACIÓN

Y FORMACIÓN DE PROFESORES

emociones experimentadas. Es por eso que el trabajo del relato escrito, así como del oral, bajo esta propuesta, puede generar la aparición del pudor y temor al juicio. Al respecto dos docentes participantes señalan respectivamente:

a mí lo que me pasó es que yo me resistía a leer mi relato, principalmente porque mostraba un conflicto que se produjo en la sala de clases. Y me resistí a leerlo la vez anterior. Cuando me di cuenta que había que leerlo dije: mejor hubiera escrito otra cosa.

[...] es súper difícil expresar situaciones complicadas, conflictivas porque, tú lo dijiste, o sea a uno lo enjuician y uno se enjuicia a sí mismo también, se culpa.

Por otro lado, también emergen relatos sobre la satisfacción de cerrar situaciones pendientes, lo que implica la reconciliación con la propia historia, una suerte de sanación. Al respecto, se señala:

la práctica del Taller anterior hizo que yo considerara este espacio como sanador, de cuidado.

El juicio es siempre rehuido, pero el ambiente que ofrece una comunidad de escucha y sostén mutuo neutraliza el temor. Los relatos se presentan en su conjunto como análogos a las propias experiencias, lo que se traduce en empatía.

Claramente hay un componente terapéutico asociado al acto de escribir, cercano a la metáfora del exorcismo, del conjuro, como lo expresa Cortázar (1994) en relación a su práctica narrativa. Por su parte, la expresión corporal comparte dicho componente terapéutico, permitiendo el juego escénico, soltar, liberar las tensiones que genera el relato.

En el Taller se trabaja en pos de la articulación de dos lenguajes y dos experiencias que permiten dar cuenta, de modo diferente, de la reflexividad como configuradora de la profesionalidad docente (Desgagné, 2005). En las sesiones, la experiencia corporal aparece asociada a la experiencia narrativa como un medio para "alivianar" el relato. Se observa una relación directa entre la emergencia del relato duro y su apaciguamiento a través de la experiencia corporal. Esta le da amplitud a la experiencia relatada, la resignifica, le da nuevos sentidos. No obstante, también existe en un registro opuesto, un reconocimiento de la experiencia corporal como opresora, del cuerpo como lugar de sometimiento, en la medida en que no permite, porque no se sabe cómo hacerlo, expresar el malestar. En un registro inicial, el "grito" se invisibiliza, desaparece. Frente a ello se propone un ejercicio orientado a despertar la conciencia del poder que otros -personas, situaciones- ejercen sobre nuestros cuerpos. Este ejercicio, entendido como un juego escénico de roles, resulta en una propuesta de re-situacionalidad en otros roles, con sus emociones y sus problemas propios. Se trabaja en el lugar del "otro" desde el cual se despierta una cierta empatía. Al finalizarlo, se realiza una puesta en común de lo vivido como experiencia corporal al haberse situado en roles que habitualmente no desempeñamos.

\subsubsection{Reflexión corporeizada: el ejercicio de la mano en la cabeza}

El trabajo corporal continúa, posterior a este ejercicio, a modo de sensibilización de los sujetos para ponerse a la escucha del cuerpo. Lo que aparentemente emerge en esta etapa del 
Taller lo llamamos "reflexión corporeizada". Se trata de una forma de reflexión que emerge como un relato encarnado, no verbalizado, sobre la vivencia de un espacio propio, cómodo, en la comunidad, un espacio de pertenencia. Esta noción pareciera apropiada para describir y teorizar sobre lo vivido con este ejercicio. Con el relato, la reflexión surge de un proceso cognitivo; con la corporalidad, la reflexión surge del movimiento y la escenificación en un juego de roles. Al respecto, surgen interrogantes emergentes sobre si hay un relato encarnado, si el cuerpo tiene un relato, la narrativa que emerge del relato corporeizado, ¿a qué corresponde? ¿Es acaso la verbalización la culminación de la reflexividad que se inicia en la experiencia corporal o es ésta otra dimensión de dicha reflexividad? Sin duda, preguntas que deberán ser abordadas en posteriores estudios.

Se observa desde el Taller una direccionalidad del ejercicio corporal en función de la formación docente. Es decir, una búsqueda de una disposición nueva y consciente de los participantes frente a sus experiencias cotidianas. La etapa de retroalimentación es clave para explorar la noción de "reflexión corporeizada".

En el inicio del ejercicio corporal, el apresto vuelve a tomar un lugar. El gesto de la mano sobre el rostro de un compañero podía despertar diversas reacciones. Para algunos fue la opresión, para otros, la conducción y aún para otro grupo fue la disposición en complicidad. La disposición a dejarse conducir evidencia la confianza como base de una buena experiencia al ser liderado. El poder no se muestra como un problema cuando media la confianza. Dicho de otro modo, el poder se presenta como un problema cuando aparece en mi cabeza como experiencia de opresión. Si no es el caso, el ser liderado y responder a dicho liderazgo, puede resultar en una experiencia incluso placentera.

El trabajo continúa en una propuesta de juego escénico. Este ejercicio no resulta para todos en una experiencia grata o al menos clara. El juego escénico, asumiendo roles, genera sorpresa, a veces angustia, y de todo ello se toma conciencia en la puesta en común, en la narrativa oral y escrita.

El ejercicio de roles despierta la empatía frente a los roles habitualmente no vividos, roles que tienen rostros y nombres. Aquí, en la puesta en común y a través del relato oral, la empatía es significada y enunciada y sin embargo, había sido sentida, corporeizada, en la situacionalidad del juego.

Sobre lo anterior, se puede observar cómo la corporalidad construye subjetividad. Inicialmente esta no es mirada ni reconocida, porque no forma parte de los lenguajes académicos o racionales. Al preguntar por lo que siente el cuerpo, se produce un silencio, tal vez un quiebre.

Situando el análisis en el ejercicio de la narrativa, inicialmente se entregan indicaciones en torno a la construcción de un relato. Básicamente se habla de sistematización y consecuentemente de una ampliación del registro. Lo que se observa es que la solicitud de trabajo sobre el relato es también una solicitud de acción. Es en el acto de la escritura que se va haciendo más presente lo vivido. Un acto que no está exento de situación, de interacción y de experiencia. Es decir, el relato permite la toma de conciencia del cuerpo sometido. El cuerpo y la palabra se potencian mutuamente, ampliando la experiencia, resignificándola, denunciando el sometimiento.

El Taller continúa en un ir y venir de la reflexión verbalizada a la reflexión corporeizada, donde la gestualidad es reinterpretada, provocando el lenguaje corporal de alguien, cierres o aperturas en quienes lo perciben. Luego, se dan indicaciones para restituir en los propios relatos la voz de los otros actores no considerados en la fase inicial. La idea es promover el despliegue empático, ya trabajado en el ejercicio corporal. 
El principio del trabajo con el relato escrito es el de la apertura de la perspectiva. En esta parte del Taller lo que se busca es abrir la experiencia y la conciencia de la misma para generar nuevas miradas sobre los problemas que han sido enunciados. Mientras que el saber se manifiesta como expresión de la experiencia sistematizada, corporalidad y narrativa se presentan como prácticas de sistematización y, en consecuencia, generadoras de saberes. Ejemplos son las nociones de poder y de liderazgo, y en el saber que ha sido construido desde la experiencia que asocia el liderazgo a un poder opresor y no a un poder conductor. Este saber y su toma de conciencia surgen como resultado de la reflexión corporeizada. Aquí se está lejos de la noción de liderazgo distribuido. Aparentemente no existe, bajo la experiencia de los docentes participantes, la corresponsabilidad en el liderazgo. Aparece, entonces, otro eco de reflexión corporeizada: seguir la orden sin cuestionar, sin pensar.

Pero el ejercicio despierta contradicciones. Lo vemos en el caso de una profesora que siente placer en ejercer el poder sobre otro, pero, conscientemente, lo rechaza ¿por qué este rechazo? ¿Será acaso que persiste la imagen socializada en el grupo, del poder del líder como un poder subyugador? ¿Cómo transformar dicha noción a favor de un liderazgo distribuido, o más bien compartido?

Por último, surge una conciencia y un saber de la contrastación entre lo vivido en el trabajo y lo experimentado por el juego escénico: se enuncia una necesidad de abrir los espacios de trabajo a la afectividad, entendida esta última como expresión de subjetividad.

\subsubsection{La experiencia del Teatro Foro}

La precariedad laboral del sistema público de educación, la particularidad con que cada docente la vivencia y las decisiones que cada uno va tomando frente a ella transversaliza la conversación en el Taller.

En la quinta sesión se plantea un trabajo colectivo basado en el Teatro-Foro donde el Grupo Interdisciplinario ha creado y preparado, previamente, una representación basada en una situación cotidiana de la vida escolar relacionada con la temática de la precariedad laboral, a partir de los testimonios y relatos expresados por los docentes en sesiones anteriores. El Teatro-Foro es una figura particular del Teatro del Oprimido desarrollado por Boal en la década de 1960. Aquí se encuentran el cuerpo-emoción, el cuerpo-relato, en una experiencia de sentidos difuminados en la acción, con la narración-cognición, la narraciónconciencia que discursea desde la emoción y la razón abriendo significados, ampliando perspectivas y transformando las disposiciones de acción. En este sentido, el ejercicio de representar un caso pedagógico de violencia escolar se convierte en un momento clave de la propuesta al confluir el interés profesional y la síntesis colectiva.

Luego de la representación, los docentes hacen un trabajo grupal identificando los problemas y piensan posibles soluciones sobre la base de la pregunta ¿qué harían ustedes en ese caso para transformar la realidad?

En las intervenciones grupales sobre este ejercicio emerge como temática central: el problema de la profesionalidad docente. En el primer grupo, las reflexiones transitan en torno a tres temas centrales: los "poderes" que intervienen en las instituciones escolares; las tensiones y contradicciones a las cuales están sometidos constantemente los profesores; y el rol docente, en el sentido de las expectativas que sobre este se tienen en lo profesional. Por último, se aborda un "problema histórico" relacionado con el componente político de la tarea docente. Al decir de uno de los participantes: 
sin poder, ni de organización, ni de articularse políticamente en comunidades... están a la deriva en un sistema que los tiene aprisionados.

Por otro lado, en el segundo grupo de trabajo también emerge la discusión sobre la "profesionalidad docente", focalizando en la dualidad entre "ser docente" y "ser humano". Una de las participantes señala:

viéndolo ni siquiera desde el lado profesional, sino de lado de las relaciones entre personas, cómo uno va a pasar por alto lo que sucede con la vida de esa persona. De repente yo creo que el rol del profesor más que enseñar un contenido, en el contexto actual, tiene que ver de pronto con sostener muchas emociones que tienen los chicos.

Desde la problematización en torno a esta dualidad surge "lo corporal" como parte importante del ejercicio profesional:

me he dado cuenta que el contacto corporal es súper fuerte, es súper potente para tener una buena relación con los alumnos, una relación cercana. A mí por lo menos eso me ha funcionado bastante y siempre trato que sea así.

En las reflexiones que hace el tercer grupo de profesores, nuevamente se aborda el problema de los "poderes" como una presión que se ejerce desde la figura del sostenedor, o cualquier otra, y la importancia de ver cómo se pronuncia cada uno en esas tensiones. Con mayor fuerza aparece la pregunta por el grado de intervención que debe tener un docente en la vida personal de sus estudiantes como parte de su "profesionalidad". Se genera entonces una controversia en relación a la realidad que viven los docentes cotidianamente en sus lugares de trabajo y el problema de los principios profesionales puestos en juego. Como señala una participante del grupo:

A veces te dicen que no hagas denuncia que es más conveniente una constancia. Pero yo creo que uno tiene que hacerlo. Para mí ni siquiera es un deber, es algo de principio interno de proteger a mis alumnos como mis hijos. (...) No porque vaya a perder un trabajo... mejor dejo pasar las cosas. Yo no soy súper profe, simplemente soy una persona que tiene una conciencia que va más allá de lo que yo pueda manejar. Para mí es un principio de que tengo que hacerlo, no puedo obviar lo evidente.

En diálogo con esta postura, otra participante del Taller ofrece una problematización histórica para abordar la disyuntiva:

muchas veces que nosotros asumamos un rol, conscientemente, con decisión, con cariño incluso, tiene que ver con un rol histórico, cultural, que el profesor asumió 150 años previo a nosotros de que nos compete la protección de los niños. $Y$ me parece que eso no es algo natural ni evidente. Insisto, uno puede acoger esa visión, y todos lo hacemos con mucho cariño, pero no es natural.

Frente a la disyuntiva ¿intervenir o no en la vida de los estudiantes? aparece también la imagen del profesor que duda o simplemente se abstiene, como lo propone otra participante 
Estudios Pedagógicos XLII, $\mathrm{N}^{\circ}$ 4: 223-242, 2016

CORPORALIDADES Y NARRATIVAS DOCENTES: UN DISPOSITIVO METODOLÓGICO PARA LA INVESTIGACIÓN Y FORMACIÓN DE PROFESORES

del grupo:

¿alimento a mis hijos o soy heroína? Y ante esa situación a veces una es egoísta y prefiere alimentar a sus hijos.... entonces no podría criticar $100 \%$ al profe que pensó en la pega porque es verdad, somos profesores, pero no somos mártires, ni monjas ni curas, y no tenemos un brazo protector detrás.

Una reflexión en torno a esta disyuntiva la realiza otra participante del Taller quien propone no generar una división entre lo que se es profesional y humanamente. Al respecto señala:

dónde nos lleva esta discusión, porque generó un conflicto este ejercicio... es importante qué tipo de profesionales y qué tipo de docentes queremos ser... Yo personalmente me lo pregunto a diario... en mi relación que tengo con el cuerpo, que es súper complejo... yo entiendo que tenemos que pagar las cuentas, pero me rehúso profundamente a debilitar mis convicciones.

Otras apelaciones al punto de quiebre que se genera con esta disyuntiva profesional son si seguir siendo profesor o salir del "sistema". En este sentido, la convicción que se tiene al respecto es sindicada como una "utopía". Al respecto, uno de los docentes manifiesta:

me molesta ver estas situaciones utópicas... siento que la realidad es mucho más compleja... A veces uno sublima la realidad para darle sentido y no perder la esperanza, pero yo creo que como profesores estamos llenos de fantasías.

Frente a esta declaración, surgen respuestas relacionadas con el sentido del trabajo docente:

para mí la utopía no tiene un rol enajenante, no es un calmante, para mí es una orientación... pero no solo eso, la idea sería que esa utopía nos lleve a pensar qué acciones necesitamos, qué tenemos que hacer para llegar a ella o simplemente, de la transformación de ésta [...] yo siento realmente que cuando uno cree en algo, tiene esa convicción de algo, de soñar un poco, yo creo que la gente cambia algo. No importa, a lo mejor un mínimo grano de arena el hecho de que uno haga ese aporte.

En síntesis, en esta sesión se concentran las dualidades presentes en el ejercicio de la docencia, especialmente el "ser" y el "rol" como si fueran algo separado y distinto. Aparece el docente desdoblado, dislocado, descorporeizado. La teatralización de la cotidianeidad de una escuela ofrece la oportunidad de evidenciarlo y hacerse cargo. Representar la escena del caso particular hace revivir las emociones, con el cuerpo cargado, la palabra liberada, y se puede personificar a quién habitualmente es el "contendor" en la escena de la escuela, el inspector, la directora, el profesor que no quiero ser pero que la realidad me obliga a ser. Se produce el efecto espejo. Se asume naturalmente en el lenguaje la separación entre el ser y la función, aunque no en todos. La desolación es el escenario para cada una de las dramatizaciones: algunos intervienen la escena denunciando a gritos como padecen aquella soledad, otros lo hacen planteando nuevos vínculos posibles por 
medio de la acogida y la comprensión. En este último plano van apareciendo los saberes docentes, en forma de aprendizajes, desde las experiencias cotidianas en la realidad de los establecimientos. Se expresa como un "saber moverse" con el cuerpo. Al decir de uno de los docentes participantes:

cariñosear con los estudiantes significa cultivar una buena relación con ellos... eso me ha funcionado bastante y siempre trato que sea así...ser versátil (psicólogo, entrenador coaching, papá y mamá, cobijo, orientador, etc.).

La conciencia de "vivir siempre bombardeados" y enfrentados diariamente a situaciones de violencia vinculadas con lo que viven sus estudiantes en sus casas, o las amenazas externas personificadas en autoridades, constituyen saberes tensionados por la pregunta acerca de qué tipo de profesional se quiere ser y el rol que se asume al respecto. Esto, en el marco de visiones hegemónicas construidas históricamente en forma exógena pero que se han interiorizado como una dimensión ética del trabajo, expresadas en ideas como "querer a los estudiantes" o "querer salvarlos", pero también como conciencia colectiva de que la precarización de los lugares de trabajo docente es producto de una historia y no de algo natural, por lo tanto, una conciencia ética también de una capacidad de cambio.

No hay salidas evidentes a la pregunta por la separación aparente entre el ser y el rol docente, sin embargo las reflexiones tienden a re-unificar estas dos dimensiones en términos de preguntar por la subjetividad docente, configurada por el malestar, por un lado, pero también por la conciencia de la utopía, del cambio. A partir de esto se abre la necesidad de plantearse, en palabras de uno de los participantes:

desde dónde estamos parados y para dónde queremos caminar, cómo queremos caminar.

Sociabilizar la experiencia docente desde lo narrativo, sería ponerle palabras al sentir. Por cierto que lo que se ha generado, tras este Taller, es una transformación personal que ha sido favorecida por la experiencia colectiva. Lo importante es destacar que por dicha socialización se pasa de constituir subjetivamente experiencias cotidianas ordinarias a constituir intersubjetivamente experiencias extraordinarias, y con ello la construcción de saberes colectivos. En efecto, uno de los últimos juegos escénicos desarrollados por los docentes participantes, expresa con gran potencia la conciencia que este grupo de docentes va adquiriendo sobre ellos mismos, sus experiencias extraordinarias y su subjetividad:

Llevamos en las mochilas el frío que se coló por las ventanas rotas de nuestros establecimientos.

\section{DISCUSIÓN Y CONSIDERACIONES FINALES}

La puesta en marcha del dispositivo Taller de Corporalidad y Narrativa Docente significó enfrentar al desafío de explorar en la experiencia subjetiva de profesores y profesoras a través de una propuesta de trabajo colaborativo entre pares, cuyo alcance no era fácil de entrever. Los diagnósticos sobre la vivencia subjetiva de docentes en el contexto y condi- 
ciones de su trabajo que evidenciaban las investigaciones referenciadas en el corpus de este trabajo, evidencian un panorama poco alentador: un/a docente emocional y corporalmente agotado/a, distanciado/a de su trabajo, con dificultad para establecer vínculos emocionales explícitos, silenciado/a o auto-silenciado/a, entre otros.

A medida que avanzaban las sesiones, y al alero de los ejercicios de activación corporal y de escritura, se iba haciendo explícito en los participantes algunos de los elementos de ese diagnóstico previo y también otro tipo de manifestaciones vividas en la experiencia subjetiva docente: cierta inhibición y rigidez corporal, inquietud y malestar ante el distanciamiento emocional impuesto desde las lógicas institucionales que deslegitiman la expresión corporal como manifestación vinculante en la relación entre docentes y estudiantes, la dificultad o ausencia de instancias institucionales para expresar la, a ratos, cruda realidad de los estudiantes (niños y jóvenes), todo lo cual se va tornando en un malestar creciente que no encuentra, en los espacios de la institución escolar, expresión o salida.

Frente al distanciamiento emocional forzado, que permite a los docentes mantenerse en el desarrollo de su labor, pero que niega el contexto emocional entre estudiantes y docentes, emerge el trabajo corporal como una herramienta privilegiada para ensayar el colocarse en otro lugar respecto a la propia vivencia y respecto a los demás. Se trata de un trabajo corporal que, sin entrar en dispositivos terapéuticos, moviliza emociones y disposiciones, destraba y permite la emergencia del pensar situado.

De un modo similar, la indagación narrativa, tanto en su dimensión oral y escritural, como en el plano individual y colectivo, constituyó, en el desarrollo del Taller una herramienta importante de indagación de la experiencia docente, emergiendo desde allí vivencias diversas, marcadas por la diferenciación de contextos vividos. No obstante la diversidad de contextos en que se situaba la vivencia relatada, fue posible dialogar en torno a problemas, situaciones, padecimientos o conflictos comunes, lo que se considera un aporte de este trabajo a los estudios sobre la narrativa docente, especialmente en su forma escrita, al visibilizar los significados y sentidos que los participantes otorgan a la experiencia vivida en un contexto colectivo, trascendiendo sus particularidades y concretitud, constituyendo el relato escrito un vehículo para tomar distancia de estas y así convertirlas en un objeto de reflexión y una vía hacia el cambio (McEwan y Egan, 2005).

Por su parte, a través de la activación del cuerpo emerge en los docentes la palabra fluida, sin preámbulos, sin la mediatez de la conciencia verbal discursiva. De este modo, se puede concluir sobre la emergencia de una ampliación de la experiencia docente en el Taller: se habla del cuerpo docente, a través del cuerpo, en complicidad a ratos tensionada con la palabra. El cuerpo habla de sus tensiones en tanto se es docente, desde la visión de una realidad personal y de relaciones fragmentadas, controladas y vigiladas en el ámbito de su trabajo. Pero el/la docente, en tanto cuerpo, también imagina, expresa y amplía la experiencia, no solo en el sentido de complejizarla, sino de expandirla en el tiempo, prefigurando nuevas posibilidades de ser y de estar más pleno en el ámbito de su quehacer y de su trabajo. Al relevarse en el relato de los participantes la dimensión corporal en la configuración de la subjetividad docente, este estudio permite ampliar las posibilidades de reconocimiento de la complejidad del ser y subjetividad docente, abarcando de modo inicial dimensiones del saber docente poco exploradas en la investigación existente a nivel nacional, posibilitando con ello reflexionar ¿de qué forma comprende y actúa en el mundo escolar y educativo un profesor? Y ¿cómo comunica lo que sabe y aprende? 
El cuerpo, en esta experiencia individual-colectiva, aparece como mediador, catalizador y atenuante del dolor y/o "malestar docente". La conciencia corporal podría eventualmente posibilitar la transformación del padecimiento en acción propositiva.

Cuerpo y palabra se potencian, dialogan y se tensionan en el espacio íntimo e individual, colectivo y público, un espacio en el que se manifiesta la expresión libre y creadora. Desde aquí se avizora la posibilidad de re-integración del sujeto docente escindido y fraccionado, formando parte y tomando el poder en la conducción y toma de conciencia de los procesos pedagógicos de los cuales día a día se hace cargo.

Una evidencia que constituye uno de los aprendizajes centrales en esta experiencia compartida es que la transformación de los ambientes escolares emerge de las posibilidades de transformación de los sujetos a partir de la toma de conciencia de su situación, de su propia capacidad y poder transformador.

Finalmente, desde lo que al Grupo deja esta relevante experiencia en términos de rescatar y relevar los saberes que se gestan en la vivencia educativa y que pone en acción diversas facultades humanas (no solo las activas o intelectivas), creemos importante comenzar a abrir un flanco para el diálogo con otras disciplinas y enfoques de conocimiento, por ejemplo, la biología y las neurociencias, que permita, desde una mayor interdisciplinariedad, abordar los temas del cuerpo, las emociones y la cognición en la construcción de la experiencia y del saber docente.

\section{REFERENCIAS BIBLIOGRÁFICAS}

Assaél, J., Cornejo, R., González, J., Redondo, J., Sánchez, R., y Sobarzo, M. (2011). La empresa educativa chilena. Educación y sociedad, 32, 305-322.

Beatrice Ávalos (2013). ¿Héroes o Villanos? La profesión docente en Chile. Santiago: Editorial Universitaria.

Bajtin, M. (1995). Estética de la creación verbal. Trad. Tatiana Bubnova, México: Siglo XXI.

Bánovčanová, Z. y Slavík, J. (2014). Body in education. Journal of Pedagogy, 5(1), Curriculum Perspectives and Shaping the Lived Body, 5 -10.

Bravo, D., Peirano, C. y Flack, D. (2006). Encuesta longitudinal de docentes 2005: análisis y principales resultados. Santiago: Centro de Microdatos, Departamento de Economía, Universidad de Chile. Ministerio de Educación.

Bruner, J. (1991). Actos de significado: Más allá de la revolución cognitiva. Madrid: Editorial Alianza.

Butler, J. (2007). El género en disputa. España: Paidos Iberoamérica.

Casassus, J. (2003). La escuela y la (des)igualdad. Santiago: LOM.

Coll, C.; Marchesi, A. y Palacios, J. (2001). Desarrollo Psicológico y Educación. Volumen II: Psicología de la educación escolar. Madrid: Alianza, 2a. ed.

Connelly, F. \& Clandinin, D. (1994). Telling teaching stories. Teacher Education Quarterly, 21(1), 145-158.

Cornejo, R. \& Reyes, L. (2008). La cuestión docente. Chile: Experiencias organizacionales y acción colectiva de profesores. Santiago, Chile: FLAPE.

Cortázar, J. (1994). Algunos aspectos del cuento. En Obra Crítica/2, Edición de Jaime Alazraki (pp. 364-385). Madrid: Alfaguara.

Desgagné, S. (1997). Le concept de recherche collaborative: l'idée d'un rapprochement entre chercheurs universitaires et praticiens enseignants. Revue des sciences de l'éducation, 23(2), 371393. 
Estudios Pedagógicos XLII, $\mathrm{N}^{\circ}$ 4: 223-242, 2016

CORPORALIDADES Y NARRATIVAS DOCENTES: UN DISPOSITIVO METODOLÓGICO PARA LA INVESTIGACIÓN

Y FORMACIÓN DE PROFESORES

Desgagné, S., Bednarz, N., Couture, C., Poirier, L., \& Lebuis, P. (2001). L'approche collaborative de recherche en éducation : un rapport nouveau à établir entre recherche et formation. Revue des sciences de l'éducation, 27 (1), [Online] Recuperado el 28 de abril, 2016 de http://www.erudit. org/revue/rse/2001/v27/n1/000305ar.html

Desgagné, S. (2005). Récits exemplaires de pratique enseignante. Analyse typologique. Québec: Presses de l'Université du Québec.

Elbaz, F. (1991). Research on Teacher's Knowledge: The Evolution of a Discourse. Journal of Curriculum Studies, 23 (1), 1-19.

Evans, J., Davies, B. y Wright, J. (eds.) (2004). Body knowledge and control. Studies in the sociology of Physical Education and Health. London: Routledge.

Fardella, C. (2012). Verdades sobre la docencia, efectos y consecuencias subjetivas de la evaluación docente en Chile. Revista de Psicología, 21(1), 209 - 227.

Fardella, C. y Sisto, V. (2015). Nuevas regulaciones del trabajo docente en Chile. Discurso, subjetividad y resistencia. Psicología y Sociedade, 27(1), 68-79.

Foucault, M. (1975). Vigilar y castigar. Nacimiento de la prisión. Buenos Aires: Siglo XXI editores.

Freud, S. (1981). Obras Completas. Madrid: Biblioteca Nueva.

Greene, M. (1995). El profesor como extranjero. En J. Larrosa, R. Arnaus, V. Ferrer, N. Pérez, F. M. Connelly, D. J. Clandinin et al. (Eds.). Déjame que te cuente: ensayos sobre narrativa y educación (pp. 81-130). Barcelona: Laertes.

Goodson, I. \&Ball, S. (Eds.) (1985). Teachers lives and careers. Londres, Nueva York y Filadelfia: Falmer.

Goodson, I. (1992). Studying Teachers' Lives - an emergent field of study. Londres: Routledge.

Hughes, B. (2000). Medicalized bodies. En Hancock, P.; Hughes, B.; Jagger, E.; Paterson, K.; Russell, R.; Tulle-Winton, E. y Tyler, M. (eds.). The body, culture and society (pp. 12-28). Philadelphia: Open University Press.

Kirk, D. (1998). Schooling bodies. School practice and public discourse 1880-1950. London: Reicester University Press.

Lacan, J. (1985). Escritos, 1 y 2. México: Siglo XXI.

Le Boulch, J. (1971). Hacia una ciencia del movimiento humano. Introducción a la psicokinética. Buenos Aires: Paidós.

Le Breton, D. (2002). La sociología del cuerpo. Buenos Aires: Nueva Visión.

Lieberman, A. \& Wood, D. (2003). Inside the National Writing Project: Connecting network learning and classroom teaching. New York: Teacher College Press.

Martínez, L. (2013). El tratamiento de lo corporal en la formación inicial del profesorado. Revista Interuniversitaria de Formación del Profesorado, 78 (27,3), 161-175.

Mclaren, P. (1994).Pedagogía crítica, resistencia cultural y la producción del deseo. Buenos Aires: Aique Editor.

Mclaren, P. (1997). Pedagogía crítica y cultura depredadora. Políticas de oposición en la era postmoderna. Barcelona: Paidós.

McWilliam, E \& Taylor, P. (eds.) (1996). Bodies, Technologies, Pedagogies. New York: Peter Lang.

Merleau-Ponty, M. (2002). Phenomenology of perception. London, New York: Routledge.

Miranda, Ch., Gysling, J., Rivera, P., Arancibia, M. \& López, P. (2015). Impacto de la Formación permanente de profesores de primaria en Chile. Evidencias para una evaluación pendiente. En Hirmas, C., Hevia, R. (Editores), Formación Permanente y Desarrollo Profesional Docente (pp. 157-177). España: OEI.

Morisse, M., Lafortune, L. \& Cros, F. (2009). Les écritures en situations professionnelles. Quebec: Presses de l'Université du Québec.

Morisse, M., Lafortune, L.\& Cros, F. (2011). Se professionnaliser par l'écriture. Quels accompagnements? Quebec: Presses de l'Université du Québec.

Márquez, M., Padua, D., Prados, E. (2015). La educación olvidó el cuerpo. De la emoción a la 
escritura. Cuadernos de Pedagogía, 453, 96-99.

Núñez, M. (2015). ¡Profesores, a escribir! Pero... ¿para qué? La escritura de relatos como un acto de resistencia a la falta de sentidos. Revista Docencia, 55, 68.

Organización para la Cooperación y el Desarrollo Económicos [OCDE] (2004). Revisión de Políticas Nacionales de Educación: Chile. París: OCDE.

Organización para la Cooperación y el Desarrollo Económicos [OCDE] (2009). Los docentes son importantes. Atraer, formar y conservar a los docentes eficientes. París: OCDE.

Parlebas, P. (1981). Contribution à un lexique commenté en science de l'action motrice. París: INSEP.

PRELAC (2003). Declaración del Proyecto Regional de Educación para Latinoamérica y el Caribe aprobada por los Ministros de Educación. La Habana: PRELAC.

Quay, J., Dickinson, A. \& Nettleton, T. (2003). Students caring for each other: outdoor education and learning through peer relationships. Australian Journal of Outdoor Education, 7(1), 45-53.

Reis, P. \& Climent, N. (2012). Narrativas de profesores: reflexiones en torno al desarrollo personal y profesional. Sevilla: Universidad Internacional de Andalucía. Recuperado el 03 de marzo, 2016 de http://dspace.unia.es/bitstream/handle/10334/2419/reis_climent_2012.pdf?sequence=3

Reyes, L.; Miranda, Ch.; Santa Cruz, E.; Cornejo, R.; Núñez, M.; Arévalo, A. \& Hidalgo, F. (2014). Subjetividad y saberes docentes en el sistema educativo chileno: un análisis desde las concepciones de formadores de profesores. Estudios Pedagógicos, 15(1), 183-203.

Rich, E. (2010). Obesity assemblages and surveillance in schools. International Journal of Qualitative Studies in Education, 23(7), 803-821.

Robalino, M. (2005). ¿Actor o protagonista?: dilemas y responsabilidades sociales de la profesión docente. Revista PRELAC, 1. Proyecto Regional de Educación para América Latina y el Caribe. UNESCO - OREALC.

Sanders, F.\& Rivers, C. (1996). Cumulative and Residual Effects of Teachers on Future Student Academic Achievement. Tennessee: University of Tennessee.

Sergio, M. (1999). Um corte epistemológico. Da educação física á motricidade humana. Lisboa: Instituto Piaget.

Suárez, D. (2007). Formación Docente e Indagación Pedagógica del Mundo Escolar. La Construcción Social del Conocimiento Escolar. Laboratorio de Políticas Públicas de Buenos Aires, UBA. Recuperado de http://www.lpp-buenosaires.net/documentacionpedagogica/ArtPon/ publicaciones_dhs/p_torres.pdf

Suárez, D. (2007b). Documentación narrativa de experiencias y viajes pedagógicos. Fascículo 2. ¿Qué es la Documentación Narrativa de Experiencias Pedagógicas? Buenos Aires: Laboratorio de Políticas Públicas de Buenos Aires, UBA.

Suárez, H. (Ed.) (2001). Expedición pedagógica nacional: huellas y registros. Encuentro Nacional de Viajeros, Armenia, Diciembre 2000. Bogotá: Universidad Pedagógica Nacional, Expedición Pedagógica Nacional, OEI, Fundación Antonio Restrepo Barco.

Turner, B. (1989). El cuerpo y la sociedad: exploraciones en teoría social. España: Fondo de Cultura Económica.

UNESCO (1990). Declaración Mundial sobre Educación para Todos y Marco de Acción para Satisfacer las Necesidades Básicas de Aprendizaje. Tailandia: UNESCO.

UNESCO (2001). Primer estudio internacional comparativo de lenguaje, matemáticas y factores asociados. Laboratorio Latinoamericano de evaluación de la calidad de la educación. Santiago de Chile: UNESCO.

UNESCO. (2008). Segundo estudio regional comparativo y explicativo: Los aprendizajes de los estudiantes de América Latina y el Caribe. Primer reporte. Laboratorio Latinoamericano de Evaluación de la Calidad de la Educación. Santiago de Chile: LLECE. UNESCO/OREALC.

Vaillant, D.(2004). Construcción de la profesión docente en América Latina. Tendencias, temas y debates. Serie Documentos PREAL, 31. Santiago.

Valdivia, G., Avendaño, C., Bastías, G., Milicic, N., Morales, A. \& Scharager, J. (2003). Estudio de 
Estudios Pedagógicos XLII, N 4: 223-242, 2016

CORPORALIDADES Y NARRATIVAS DOCENTES: UN DISPOSITIVO METODOLÓGICO PARA LA INVESTIGACIÓN

Y FORMACIÓN DE PROFESORES

la salud laboral de los profesores en Chile. Santiago: Pontificia Universidad Católica de Chile.

Vander, C. \& Gard, M. (2014). Healthy, happy and ready to teach, or why kids can't learn from fat teachers: the discursive politics of school reform and teacher health. Critical Public Health, 24(2), 210-225.

Vick, M. \& Martínez, C. (2011). Teachers and teaching: subjectivity, performativity and the body. Educational Philosophy and Theory, 43(2), 178-192. 